\title{
Transection of the cervical sympathetic trunk inhibits the progression of pulmonary arterial hypertension via ERK-1/2 Signalling
}

\author{
Yongpeng Zhao ${ }^{1}$, Rui Xiang ${ }^{1}$, Xin Peng ${ }^{1}$, Qian Dong ${ }^{1}$, Dan Li ${ }^{1}$, Guiquan Yu', Lei Xiao ${ }^{2,3}$, Shu Qin ${ }^{1}$ and Wei Huang ${ }^{1 *}$
}

\begin{abstract}
Background: Abnormal sympathetic hyperactivity has been shown to lead to pulmonary arterial hypertension (PAH) deterioration. The purpose of this study was to examine whether the transection of the cervical sympathetic trunk (TCST) can inhibit the progression of PAH in a monocrotaline (MCT)-induced PAH model and elucidate the underlying mechanisms.
\end{abstract}

Methods: Rats were randomly divided into four groups, including a control group, an MCT group, an MCT + sham group and an MCT + TCST group. After performing haemodynamic and echocardiographic measurements, the rats were sacrificed for the histological study, and the norepinephrine (NE) concentrations and protein expression level of tyrosine hydroxylase $(\mathrm{TH})$ were evaluated. The protein expression levels of extracellular signal-regulated kinase (ERK)-1/2, proliferating cell nuclear antigen (PCNA), cyclin A2 and cyclin D1 in pulmonary artery vessels and pulmonary arterial smooth muscle cells (PASMCs) were determined.

Results: Compared with the MCT + sham group, TCST profoundly reduced the mean pulmonary arterial pressure (mPAP) $(22.02 \pm 4.03 \mathrm{mmHg}$ vs. $31.71 \pm 2.94 \mathrm{mmHg})$, right ventricular systolic pressure (RVSP) $(35.21 \pm 5.59 \mathrm{mmHg}$ vs. $48.36 \pm 5.44 \mathrm{mmHg}$ ), medial wall thickness (WT\%) $(22.48 \pm 1.75 \%$ vs. $46.10 \pm 3.16 \%)$, and right ventricular transverse diameter (RVTD) $(3.78 \pm 0.40 \mathrm{~mm}$ vs. $4.36 \pm 0.29 \mathrm{~mm})$ and increased the tricuspid annular plane systolic excursion (TAPSE) $(2.00 \pm 0.12 \mathrm{~mm}$ vs. $1.41 \pm 0.24 \mathrm{~mm}$ ) (all $P<0.05)$. The NE concentrations and protein expression levels of TH were increased in the PAH rats but significantly decreased after TCST. Furthermore, TCST reduced the increased protein expression of PCNA, cyclin A2 and cyclin D1 induced by MCT in vivo. We also found that NE promoted PASMC viability and activated the ERK-1/2 pathway. However, the abovementioned NE-induced changes could be suppressed by the specific ERK-1/2 inhibitor U0126.

Conclusion: TCST can suppress pulmonary artery remodelling and right heart failure in MCT-induced PAH. The main mechanism may be that TCST decreases the NE concentrations in lung tissues, thereby preventing NE from promoting PASMC proliferation mediated by the ERK-1/2 signalling pathway.

Keywords: Transection of the cervical sympathetic trunk, Sympathetic nerve block, Pulmonary arterial hypertension

\footnotetext{
* Correspondence: weihuangcq@gmail.com

${ }^{1}$ Department of Cardiology, the First Affiliated Hospital of Chongqing

Medical University, 1 Youyi Road, Yuzhong District, Chongqing, China

Full list of author information is available at the end of the article
}

(c) The Author(s). 2019 Open Access This article is distributed under the terms of the Creative Commons Attribution 4.0 International License (http://creativecommons.org/licenses/by/4.0/), which permits unrestricted use, distribution, and reproduction in any medium, provided you give appropriate credit to the original author(s) and the source, provide a link to the Creative Commons license, and indicate if changes were made. The Creative Commons Public Domain Dedication waiver (http://creativecommons.org/publicdomain/zero/1.0/) applies to the data made available in this article, unless otherwise stated. 


\section{Background}

Pulmonary arterial hypertension (PAH) is a progressive disease, defined as an increase in the mean pulmonary arterial pressure (mPAP) $\geq 25 \mathrm{mmHg}$ at rest as assessed by right heart catheterization and is associated with a poor prognosis [1]. This disease shares the following common pathophysiological and histological features: pathologic pulmonary vasoconstriction, remodelling of the small pulmonary arteries and thrombosis [2, 3]. These pathological changes contribute to increased pulmonary vascular resistance, ultimately leading to right ventricular (RV) failure and death. While many advances in therapies for PAH have been achieved, the survival rate remains poor (the 1 - and 5-year survival rates are 86.3 and $61.2 \%$, respectively) $[4,5]$.

Over the past two decades, accumulating evidence has suggested that PAH is generally associated with increased sympathetic nervous system activation [6, 7]. In addition, excess sympathetic activation may be an independent predictor of clinical deterioration [7-9]. Therefore, in addition to pharmacological therapy, different treatments, such as renal denervation $[10,11]$ and pulmonary artery denervation (PADN) [12-15], have been considered to reduce sympathetic activity and improve $\mathrm{PAH}$. Although renal denervation and PADN reportedly decrease mPAP and prevent the progression of PAH in experimental and clinical trials, the mechanism by which denervation acts in the treatment of PAH remains largely unclear. Moreover, there are several limitations to artery denervation as follows: 1) catheter-based radiofrequency denervation of the arterial sympathetic nerves may lead to arterial stenosis; 2) there is no direct measure which can confirm that the renal or pulmonary artery nerves have in fact been denervated; and 3) the denervation procedure may injure vascular parasympathetic nerves. Therefore, investigating new sympathetic blocking methods for the treatment of $\mathrm{PAH}$ is essential.

Cervical sympathetic and stellate ganglion blocks (SGBs) have been used for the treatment of several cardiovascular diseases [16-18]. These procedures inhibit sympathetic activity by decreasing efferent cervical and upper thoracic sympathetic outflow, and successful sympathetic blockade can easily be recognized by the presence of Horner's syndrome [19]. The transection of the cervical sympathetic trunk (TCST) is a procedure leading to long-term and repeated SGBs. Additionally, pulmonary sympathetic innervation mainly originates from the middle and inferior cervical and thoracic sympathetic chain (T2 to T8), and the pulmonary vasculature is the major sympathetic target in the lung [20]. Since most pulmonary sympathetic axons release norepinephrine (NE), we hypothesized that TCST could decrease the production of $\mathrm{NE}$ in the lung.
The proliferation of pulmonary artery smooth muscle cells (PASMCs) has been documented to play a critical role during the progression of $\mathrm{PAH}$ [3]. Previous studies have demonstrated that NE induces proliferation in PASMCs [21] and contributes to pulmonary vascular remodelling [22]. Moreover, the extracellular signalregulated kinase $1 / 2$ (ERK-1/2) signalling pathway plays an important role in multiple cellular functions, including differentiation, proliferation and migration [23]. Evidence suggests that the aberrant activation of ERK-1/2 may lead to increased proliferation in PASMCs [21, 24]. Based on the above findings, we hypothesized that TCST could inhibit the progression of $\mathrm{PAH}$. Accordingly, the aims of the present study were to explore 1) whether haemodynamic and pulmonary artery remodelling could be improved by TCST in an MCT-induced PAH model in rats and 2) whether the ERK-1/2 pathway participates in the NE-induced PASMCs proliferation.

\section{Methods \\ Materials}

Monocrotaline (MCT, NO. M0418-500), NE (NO.M8550) and prazosin (Pra, NO.MT1050) were provided by Mengbo (Chongqing, China). U0126 (NO. NY12031) and Cell Counting Kit-8 (CCK-8, NO. HYK0301) were obtained from MedChemExpress (MCE, NJ, USA). The NE Elisa Kit (NO. 2907A) was obtained from MB-Biology (Jiangsu, China). The anti-tyrosine hydroxylase (TH, NO.\#13,106), ERK-1/2 (NO.\#4695) and p-ERK-1/2 (NO.\#9101) antibodies were provided by Cell Signaling Technology (Beverly, MA, USA). The anti- $\alpha$ smooth muscle actin ( $\alpha$-SMA) (NO. ab5694), CD3 (NO. ab16669), cyclin A2 (NO. ab181591), and cyclin D1 (NO. ab134175) antibodies were purchased from Abcam (Cambridge, UK). The proliferating cell nuclear antigen (PCNA, NO. 10205-2-AP), $\beta$-actin (No.20536-1-AP), and GAPDH (No.10494-1-AP) antibodies and the secondary antibodies (NO.SA00001-10) were obtained from Proteintech (Wuhan, Hubei, China). All reagents were obtained from common commercial sources.

\section{Animals and experimental design}

All animal procedures were carried out in accordance with the ARRIVE guidelines and the UK Animals (Scientific Procedures) Act of 1986 and its associated guidelines. The experimental protocol was approved by the Ethics Committee of Chongqing Medical University (Chongqing, China).

The study protocol was shown in Fig. 1. Briefly, fiftyfive male Sprague-Dawley rats (aged 8 weeks, weight of 200-250 g) obtained from the Chongqing Laboratory Animal Center were randomly assigned to 4 groups. Group 1 included 10 rats that received intraperitoneal injections of normal saline (Control group). Group 2 


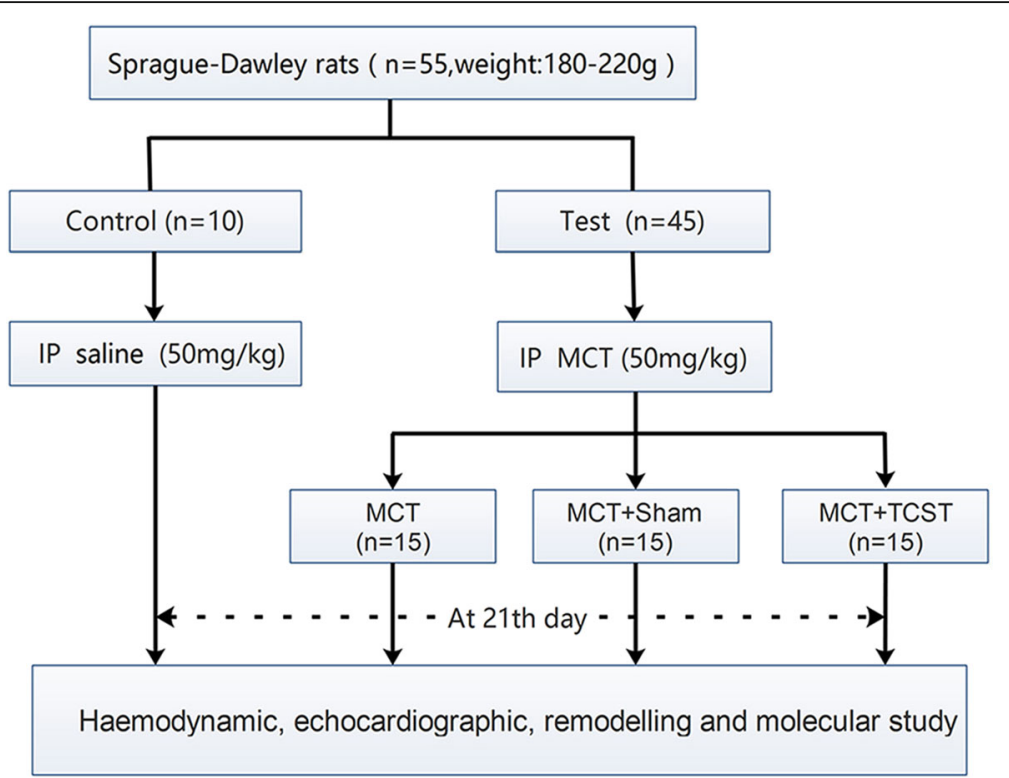

Fig. 1 Flow chart of the study. IP, intraperitoneal injection; MCT, monocrotaline; TCST, transection of the cervical sympathetic trunk

included 15 rats that received intraperitoneal injections of MCT (MCT group). Group 3 included 15 rats that received intraperitoneal injections of MCT after $1 \mathrm{~h}$ of sham TCST (MCT + sham group). Group 4 included 15 rats that received intraperitoneal injections of $\mathrm{MCT}$ after $1 \mathrm{~h}$ of TCST (MCT + TCST group). The rats had free access to food and water and were maintained in a room with controlled temperature $\left(22 \pm 2{ }^{\circ} \mathrm{C}\right)$ and lighting under 12-h light/dark exposure cycles. At the end of the study, after the haemodynamic parameters were measured and the tissues were collected, all rats were sacrificed.

\section{MCT-induced PAH}

MCT was dissolved in $1 \mathrm{~mol} / \mathrm{L} \mathrm{HCl}$, the $\mathrm{pH}$ was adjusted to 7.4, and the final concentration of MCT was adjusted to $10 \mathrm{mg} / \mathrm{ml}$ with distilled water. The MCT-induced PAH rats were intraperitoneally injected with $50 \mathrm{mg} / \mathrm{kg}$ of MCT. In the control group, saline was injected instead of MCT.

\section{Transection of the cervical sympathetic trunk (TCST)}

Before all surgeries, the rats were anaesthetized with $2 \%$ pentobarbital sodium $(30 \mathrm{mg} / \mathrm{kg}$, intraperitoneal). A 2$\mathrm{cm}$ vertical incision was created in the neck region. Then, the right sternomastoid muscle and omohyoid muscle were separated (not transected). The right common carotid artery was exposed, and the cervical sympathetic trunk was identified along the proximal side of the superior cervical ganglion [25]. The right side of the cervical sympathetic trunk was transected, and the incision was closed (Fig. 2b). In the rats in the sham group, the right common carotid artery and cervical sympathetic trunk were exposed without transection of the nerve. The sympathetic blockades were confirmed by blepharoptosis on the operated side (Fig. 2c).

\section{Echocardiographic assessment}

Two-dimensional (2D) images of the right ventricular transverse diameter (RVTD) and pulmonary artery transverse diameter (PATD) were measured on apical 4chamber and parasternal short-axis views, respectively (IE33; Philips, Holland). The left ventricular internal diameter during systole (LVIDs) and left ventricular internal diameter during diastole (LVIDd) were measured on M-mode echocardiography parasternal long-axis views. Then, the cardiac output (CO) was calculated by computer algorithms. The tricuspid annular plane systolic excursion (TAPSE) was measured from M-mode apical 4-chamber views. All data represented the mean of five uninterrupted cardiac cycles.

\section{Haemodynamic and right ventricular hypertrophy measurements}

A homemade polyethylene catheter (inner diameter: 0.5 $\mathrm{mm}$, outside diameter: $0.9 \mathrm{~mm}$ ) filled with heparin was connected to a Multi-lead Physiological Recorder (MP150, BIOPAC System, USA). Then, the catheter was inserted into the RV of the anaesthetized rats via the right jugular vein and introduced into the pulmonary artery guided by a pressure curve (Fig. 2a). After measuring the pulmonary arterial pressure, the same catheter was inserted into the carotid artery to measure the 

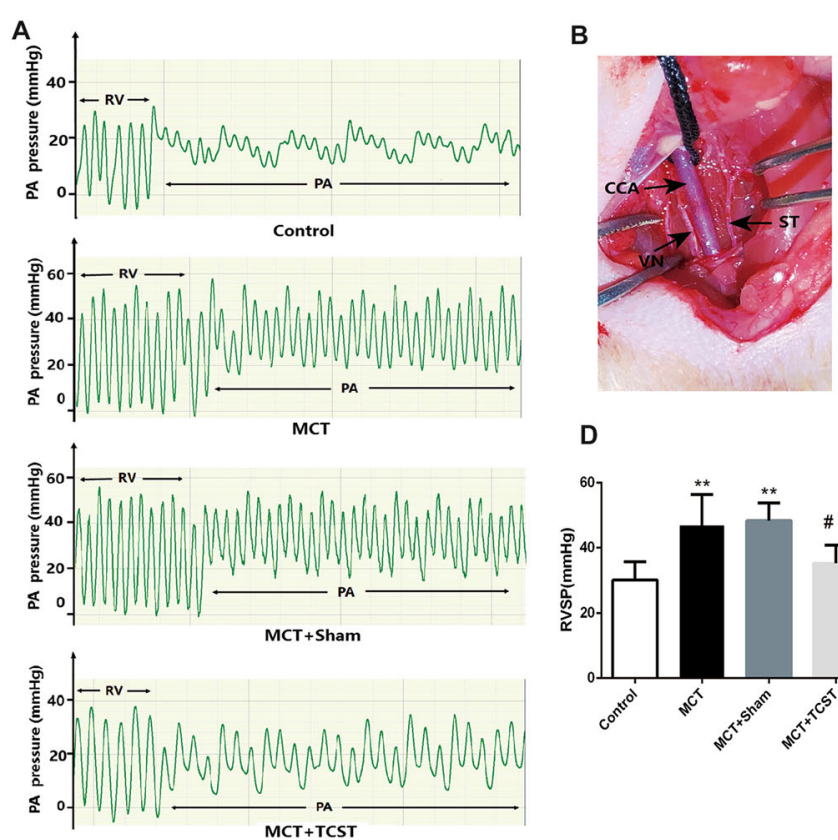

D

C

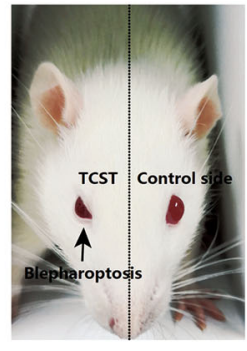

E

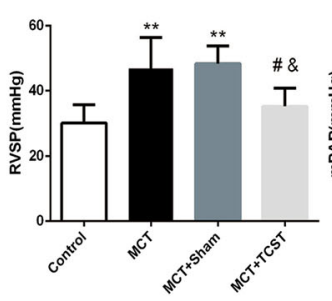

Fig. 2 Right ventricular systolic pressure (RVSP) and mean pulmonary arterial pressure (mPAP); (a) Assessment of the RVSP and mPAP in MCTinduced pulmonary arterial hypertension (PAH) rats. RV, right ventricle; PA, pulmonary artery. b Photograph of the dissection of a rat's neck. CCA, common carotid artery; VN, vagus nerve; ST, sympathetic trunk. c Unilateral blepharoptosis after TCST. $\mathbf{d}$, e The RVSP and mPAP were significantly elevated in the MCT and MCT + sham groups, whereas this effect was attenuated by TCST. ${ }^{* *} P<0.01$ vs. control group; $\# P<0.01$ vs. MCT group; $\& P<0.01$ vs. MCT + sham group

systemic arterial pressures, and the heart rate (HR) was measured.

To evaluate RV hypertrophy, the atria were removed. Then, the heart was dissected into the RV and left ventricle (LV) plus septal wall and weighed. The ratio of the weight of the RV to the weight of the LV plus septum $[\mathrm{RV} /(\mathrm{LV}+\mathrm{S})]$ was used as an index of RV hypertrophy.

\section{Histology and immunohistochemistry}

The lung tissues were obtained from anaesthetized rats and immersed in $4 \%$ paraformaldehyde overnight for fixation. The fixed tissues were dehydrated, cleared, embedded in paraffin wax, and cut into $5-\mu \mathrm{m}$ thick sections. Haematoxylin and eosin ( $\mathrm{H} \& \mathrm{E})$, Elastic-Van Gieson, $\alpha$-SMA and CD3 staining were performed on lung tissue sections.

The immunohistochemical staining procedure was performed according to a previously described method. Briefly, the tissue sections were deparaffinized and rehydrated in graduated alcohol. After the antigen retrieval, the endogenous peroxidase activity was blocked by incubation with $0.3 \%$ hydrogen peroxide at room temperature for $10 \mathrm{~min}$. The sections were blocked with $2 \%$ bovine serum albumin (BSA) in phosphate-buffered saline (PBS) and then incubated with the $\alpha$-SMA (1:500) and CD3 (1:200) antibodies overnight, followed by incubation with the secondary antibody. The primary antibody was not applied in the parallel controls. After the colour development through incubation with diaminobenzidine, the sections were counterstained with haematoxylin. The developed tissue sections were imaged under a microscope (Leica Microsystems DFC550, Germany). The CD3 positive index was counted as the percentage of positive cells divided by all lung cells at a high magnification $(\times 400)$, and at least 10 independent fields per section were assessed. The wall thickness (WT) and external diameter (ED) of the pulmonary arteries were measured using IPP 6.0 image analysis software (Media Cybernetics). Pulmonary arteriole remodelling was quantified as follows: $\mathrm{WT} \%=2 \times \mathrm{WT} /$ $\mathrm{ED} \times 100 \%$.

\section{Measurement of NE concentrations}

To examine whether MCT induced an increase in the generation of NE, an NE ELISA Kit was used to detect the concentrations of NE in the rat lung homogenate. The samples were thawed on ice, and the concentrations of NE were measured according to the manufacturer's instructions. The sensitivity of the assay was $8 \mathrm{pg} / \mathrm{ml}$.

\section{Preparation of PASMCs}

Pulmonary artery tissues were isolated from the rats. The pulmonary arteries were minced to a size of $1 \times 1$ $\mathrm{mm}$, placed in a dish, and incubated with DMEM 
(Dulbecco's modified Eagle's medium) supplemented with $20 \%$ foetal bovine serum (FBS), $1 \%$ penicillin and streptomycin in a humidified incubator with $5 \% \mathrm{CO}_{2}$ and $21 \% \mathrm{O}_{2}$ at $37^{\circ} \mathrm{C}$. The PASMCs were identified as having a smooth muscle phenotype by positive immunofluorescence with an anti- $\alpha$-SMA antibody. After 3-5 cell passages, the cells were used for the following experiments.

\section{Cell viability assay}

PASMCs $\left(1 \times 10^{4}\right.$ cells/well $)$ were seeded in 96-well plates, covered with $100 \mu \mathrm{L}$ of medium in each well for $24 \mathrm{~h}$, and subsequently exposed to different treatments in accordance with the group assignment. The cell viability was determined by a Cell Counting Kit- 8 and a microplate reader (Multiskan MK33; Thermolab Systems, Helsinki, Finland) at a wavelength of $450 \mathrm{~nm}$.

\section{EdU staining}

A Click-tm EdU Cell Proliferation Kit (NO. C0075S, Beyotime Bio, Shanghai, China) was used for the cell proliferation assay. The PASMCs were incubated with $10 \mu \mathrm{mol} / \mathrm{L}$ EdU for $2 \mathrm{~h}$ at $37^{\circ} \mathrm{C}$ after treatment. The cells were permeabilized with $0.3 \%$ Triton X-100 for $20 \mathrm{~min}$ after fixation with $4 \%$ formaldehyde for $15 \mathrm{~min}$ at room temperature. After three washes with PBS, the cells were incubated with click additive solution for $30 \mathrm{~min}$. PASMC nuclei stained with Hoechst-33,342 were used for the cell counts and examined using fluorescence microscopy. Five randomly selected views per sample image were used to calculate the relative EdU-positive ratio.

\section{Western blot analysis}

Pulmonary artery tissues separated from the lungs of rats were homogenized in ice-cold lysis buffer. The resulting homogenate was centrifuged at $12,000 \mathrm{rpm}$ for $15 \mathrm{~min}$ at $4{ }^{\circ} \mathrm{C}$. The supernatants were collected and stored at $-80^{\circ} \mathrm{C}$ for the Western blot analysis. The PASMCs were treated with different drugs according to the different experimental groups. The proteins were extracted from the treated PASMCs.

The pulmonary artery tissue homogenates and cell protein samples were separated by SDS-PAGE on a 10$12 \%$ gel (No.P0012A, RO Beyotime Bio) and transferred to a PVDF membrane. The membranes were blocked in $5 \%$ non-fat milk for $2 \mathrm{~h}$ and incubated with the primary antibodies [Beta-actin (1:5000), GAPDH (1:5000) TH (1: 1000), ERK-1/2 (1:1000), p-ERK-1/2 (1:1000), PCNA (1: 2000), cyclin A2 (1:2000), and cyclin D1 (1:10000)] overnight at $4{ }^{\circ} \mathrm{C}$. The blots were incubated with secondary antibodies (1:5000) for another $2 \mathrm{~h}$ at room temperature. The signals were examined with a Bio-Rad gel imaging system (Bio-Rad, Hercules, CA, USA) and a
WesternBright ${ }^{\text {ix }}$ MCF fluorescent Western blotting kit (Advansta, Menlo Park, USA), and the results were analysed with a gel imaging system.

\section{Statistical analysis}

All experiments were repeated at least three times. The data are reported as the group mean \pm standard deviation (SD). SPSS 22.0 (IBM Corporation, Armonk, USA) was used for the statistical analyses. The differences between the groups were determined by Student's t-tests. A $P$-value $<0.05$ was considered statistically significant.

\section{Results}

\section{Mortality and the PAH model}

Twenty-one days after the injection with MCT, in total, 10 deaths occurred, including 6 in the MCT group and 4 in the MCT + sham group. Finally, the control group remained 10 rats, the MCT group remained 9 rats, the $\mathrm{MCT}+$ sham group remained 11 rats, and the MCT + TCST group remained 15 rats.

To confirm whether the rat model of PAH established, the mPAP was measured twenty-one days following the MCT injection. Compared with the control rats, the mPAP was significantly increased (Table 1, Fig. $2 \mathrm{a}$ and e) in the rats in the MCT group, indicating the successful induction of PAH. There was no significant difference in the mean systemic arterial pressure or HR between the control rats and MCT rats (Table 1), suggesting that MCT had no impact on the systemic arterial pressure.

\section{TCST attenuated the MCT-induced increase in RVSP and mPAP}

Twenty-one days after the MCT injections, the RVSP and MPAP in the MCT and MCT + sham groups were significantly increased compared with those in the control group (Table 1, Fig. 2d and e). However, compared with the MCT + sham group, the RVSP and MPAP in the MCT + TCST group were significantly decreased (RVSP: $\quad 35.21 \pm 5.59 \mathrm{mmHg} \quad$ vs. $\quad 48.36 \pm 5.44 \mathrm{mmHg}$, mPAP: $22.02 \pm 4.03 \mathrm{mmHg}$ vs. $31.71 \pm 2.94 \mathrm{mmHg}$, respectively; both $P<0.01$; Table 1 , Fig. $2 \mathrm{~d}$ and e).

\section{Effects of TCST on RV hypertrophy and cardiac function in MCT-induced PAH}

The RVTD and RV/LV $+\mathrm{S}$ in the MCT + TCST group were significantly lower than those in the MCT + sham group (RVTD: $3.78 \pm 0.40 \mathrm{~mm}$ vs. $4.36 \pm 0.29 \mathrm{~mm}$; RV/ $\mathrm{LV}+\mathrm{S}: 0.24 \pm 0.07$ vs. $0.48 \pm 0.10$; both $P<0.01$; Table 1 , Fig. 3a, c and f), suggesting that TCST decreased RV hypertrophy in the PAH rats. Additionally, compared with the MCT + sham group, the TAPSE and CO were significantly increased in the MCT + TCST group (TAPSE: $2.00 \pm 0.12 \mathrm{~mm}$ vs. $1.41 \pm 0.24 \mathrm{~mm} ; P<0.05$; CO: $204.72 \pm 11.13 \mathrm{ml} / \mathrm{min}$ vs. $178.53 \pm 6.75 \mathrm{ml} / \mathrm{min} ; P<$ 
Table 1 Comparison of the haemodynamic data and RV function in the MCT-induced PAH rats

\begin{tabular}{lllll}
\hline & Control & MCT & MCT + sham & MCT + TCST \\
\hline HR (beats/min) & $408 \pm 39$ & $391 \pm 24$ & $407 \pm 26$ & $421 \pm 15$ \\
mSAP $(\mathrm{mmHg})$ & $108.89 \pm 6.88$ & $100.05 \pm 7.77$ & $95.08 \pm 4.84$ & $108.87 \pm 9.30$ \\
RVSP $(\mathrm{mmHg})$ & $30.85 \pm 5.32$ & $46.83 \pm 9.53^{* *}$ & $48.36 \pm 5.44^{* *}$ & $35.21 \pm 5.59^{\# \&}$ \\
mPAP $(\mathrm{mmHg})$ & $17.34 \pm 0.88$ & $34.13 \pm 1.30^{* *}$ & $31.71 \pm 2.94^{* *}$ & $22.02 \pm 4.03^{\# \&}$ \\
RVTD $(\mathrm{mm})$ & $3.69 \pm 0.25$ & $4.42 \pm 0.27^{* *}$ & $4.36 \pm 0.29^{* *}$ & $3.78 \pm 0.40^{\# \&}$ \\
PATD $(\mathrm{mm})$ & $2.33 \pm 0.23$ & $2.43 \pm 0.38$ & $2.63 \pm 0.26$ & $2.62 \pm 0.18$ \\
TAPSE $(\mathrm{mm})$ & $2.12 \pm 0.04$ & $1.34 \pm 0.10^{*}$ & $1.41 \pm 0.24^{*}$ & $2.00 \pm 0.12^{\$}$ \\
CO $(\mathrm{ml} / \mathrm{min})$ & $199.15 \pm 12.17$ & $185.78 \pm 12.88^{* *}$ & $178.53 \pm 6.75^{* *}$ & $204.72 \pm 11.13^{\# \&}$ \\
RV/LV $+\mathrm{S}$ & $0.22 \pm 0.08$ & $0.38 \pm 0.08^{* *}$ & $0.48 \pm 0.10^{* *}$ & $0.24 \pm 0.07^{\# \&}$ \\
\hline
\end{tabular}

All values are expressed as the mean $\pm \mathrm{SD}$. CO, cardiac output; HR, heart rate; $\mathrm{MPAP}$, mean pulmonary arterial pressure; RVSP, right ventricular systolic pressure; mSAP, mean systemic arterial pressure; PATD, pulmonary artery transverse diameter; RV/(LV + S), right ventricle/(left ventricle + septum); RVTD, right ventricular transverse diameter; TAPSE, tricuspid annular plane systolic excursion; ${ }^{*} P<0.05$ vs. control group; $\$ P<0.05$ vs. MCT or MCT + sham group; ${ }^{* * P}<0.01$ vs. control group; $\# P<0.01$ vs. MCT group; $\& P<0.01$ vs. $M C T+$ sham group

0.01; Table 1, Fig. 3b, d and e), demonstrating that TCST improve the right ventricular function in $\mathrm{PAH}$.

\section{TCST attenuated the medial thickening of the pulmonary artery induced by MCT}

To investigate the effects of TCST on pulmonary vascular remodelling in the MCT-induced PAH model, the morphology of the pulmonary vessels was examined by $\mathrm{H}$ \& E, Elastic-Van Gieson and immunohistochemical staining of $\alpha$-SMA. As shown in Fig. 4 (a, b, c, and e), the pulmonary artery WT\% was greatly enhanced in the MCT group compared with that in the control group $(40.21 \pm 3.70 \%$ vs. $12.20 \pm 2.23 \% ; \quad \mathrm{P}<0.01)$. Nonetheless, the WT\% was dramatically reduced in the TCST group compared to that in the MCT and MCT + sham groups $(22.48 \pm 1.75 \%$ vs. $40.21 \pm 3.70 \%$ and $46.10 \pm 3.16 \%$; both $\mathrm{P}<0.01)$. These results suggested that the rats with MCT-induced PAH exhibited exaggerated pulmonary vascular remodelling, but such remodelling was attenuated by TCST. In addition, to determine the effects of TCST on lung inflammation induced by MCT, the lung tissue sections were stained with a T-cell marker (CD3). The results showed that TCST significantly decreased MCT-induced T-cell accumulation $(51.28 \pm 3.48 \%$ in the MCT group vs. $21.56 \% \pm 2.82 \%$ in the $\mathrm{MCT}+\mathrm{TCST}$ group; $\mathrm{P}<0.01$, Fig. $4 \mathrm{~d}$ and $\mathrm{f}$ ).

\section{TCST reduced the concentrations of NE in lung tissues of $\mathrm{PAH}$ rats}

The concentrations of NE in lung tissues of MCT rats were significantly higher than that in the control group $(250.5 \pm 15.64 \mathrm{pg} / \mathrm{mL}$ vs. $173.6 \pm 13.19 \mathrm{pg} / \mathrm{mL}, \quad \mathrm{P}<0.01)$. Whereas, the concentrations of NE in MCT + TCST rats decreased significantly compared with $\mathrm{MCT}+$ sham group $(181.0 \pm 4.83 \mathrm{pg} / \mathrm{mL}$ vs. $247.1 \pm 16.91 \mathrm{pg} / \mathrm{mL} ; \quad P<$ 0.01 , Fig. 4g). Moreover, MCT significantly increased the expression of $\mathrm{TH}$ (the rate-limiting enzyme of $\mathrm{NE}$ production) compared with control group, which were inhibited by TCST treatment (Fig. 4h).

\section{TCST inhibited MCT-induced cell proliferation and increased cyclin protein expression in vivo}

To determine whether cell proliferation is inhibited by TCST, we determined the protein expression of PCNA, cyclin A2 and cyclin D1 in pulmonary artery tissues. The data showed that compared with the control group, MCT significantly increased the expression of PCNA, cyclin A2 and cyclin D1 in the MCT-induced PAH rats, whereas the expression levels of these proteins were decreased in the MCT + TCST group (Fig. 5a, b, and c). Moreover, ERK-1/2 phosphorylation in the pulmonary artery tissues was significantly increased in the MCT group compared with that in the control group but was reduced in the MCT + TCST group (Fig. $5 \mathrm{~d}$ ). These results indicated that TCST may attenuate MCT-induced PAH partially by inhibiting the ERK- $1 / 2$ pathway.

\section{NE mediated proliferation in PASMCs}

A CCK-8 assay was applied to determine the effect of NE on PASMC proliferation. A dose-dependent effect was observed by treating the PASMCs with $1 \mathrm{nM}, 10$ $\mathrm{nM}, 100 \mathrm{nM}, 1 \mu \mathrm{M}, 10 \mu \mathrm{M}$ and $100 \mu \mathrm{M}$ NE. These results show that $\mathrm{NE}$ caused a significant increase in PASMC viability at a concentration of $1 \mu \mathrm{M}$ (Fig. 5e). Additionally, we investigated the effect of Pra on NEinduced cell proliferation. The PASMCs were treated with increasing doses $(0.05 \mu \mathrm{M}, 0.1 \mu \mathrm{M}, 1 \mu \mathrm{M}, 10 \mu \mathrm{M}$, and $100 \mu \mathrm{M})$ of Pra before the NE treatment. The results showed that Pra decreased pulmonary artery smooth muscle cell proliferation at a concentration of $1 \mu \mathrm{M}$ (Fig. 5f). Based on our dose-response effects, we decided to use NE and Pra at $1-\mu \mathrm{M}$ concentration in the subsequent experiments. 


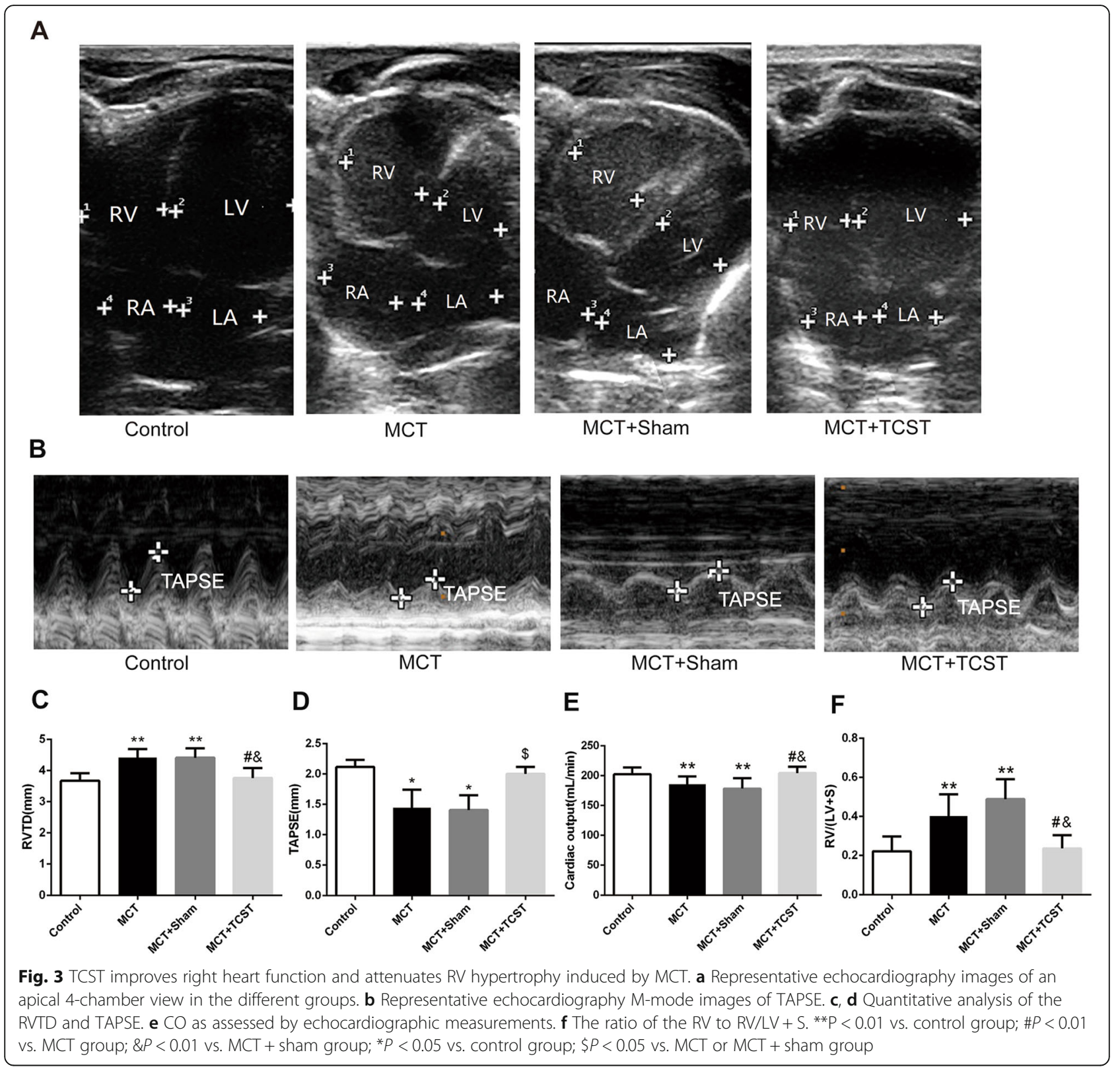

Effect of ERK-1/2 on NE-induced proliferation in PASMCs

To further elucidate whether the ERK-1/2 pathway participates in NE-mediated proliferation, we blocked ERK1/2 with U0126 and blocked NE with Pra.

As shown in Fig. 6c, NE increased the phosphorylation of ERK-1/2, and this increase was reduced by Pra or U0126, suggesting that ERK-1/2 is likely involved in NE-mediated proliferation in PASMCs. In addition, Pra decreased proliferation by almost two-fold as measured by EdU in the NEinduced PASMCs, and U0126 had a similar effect (Fig. 6a and $b$ ). Furthermore, compared with the control group, NE promoted the expression of PCNA, cyclin A2 and cyclin D1 in the PASMCs, and this increase was suppressed by the Pra or U0126 treatment (Fig. 6d, e, and f).

\section{Discussion}

The present study mainly demonstrated that TCST suppresses sympathetic activity by decreasing the NE concentrations in lung tissues, thereby attenuating pulmonary artery remodelling and RV hypertrophy in MCT-induced PAH rats. ERK activation associated with MCT was also found to be inhibited after TCST, and the inhibition of ERK activation reduced NE-induced proliferation in PASMCs. These findings may explain the link between PAH and excessive sympathetic activation, offering a potential therapy with sympathetic blocks in the treatment of PAH.

Anatomical studies have shown that the pulmonary vasculature is surrounded by adventitia, distributed 


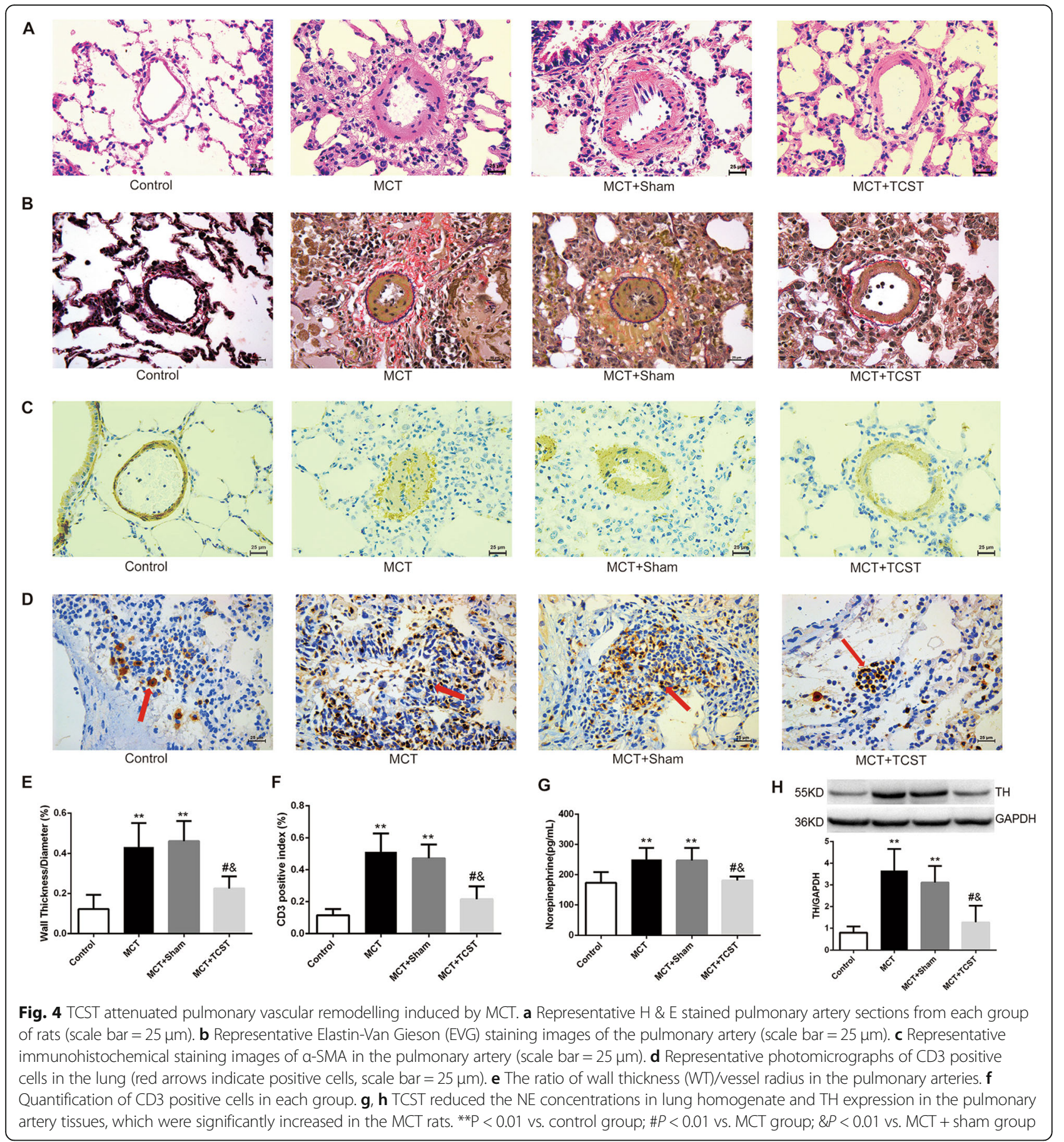

sympathetic, parasympathetic, and sensory nerve fibres [26]. The adrenergic nerves surrounding the pulmonary arteries have been visualized with fluorescence histochemistry to detect catecholamines [27, 28], and the increased vascular resistance has been shown to be mediated by $\alpha$-adrenoreceptors upon sympathetic nerve stimulation [29]. Moreover, sympathetic hyperactivity in $\mathrm{PAH}$ is evidenced by increased muscle sympathetic nerve activity and impaired HR variability [7], and this sympathetic hyperactivity has been reported to parallel the progression of PAH [8]. These findings form the theoretical basis of sympathetic blockade for the treatment of PAH. TCST had the distinct advantage of specific sympathetic denervation without adjacent parasympathetic nerve and vascular injury. Our previous studies have shown that the prognosis of rats with PAH was 
A
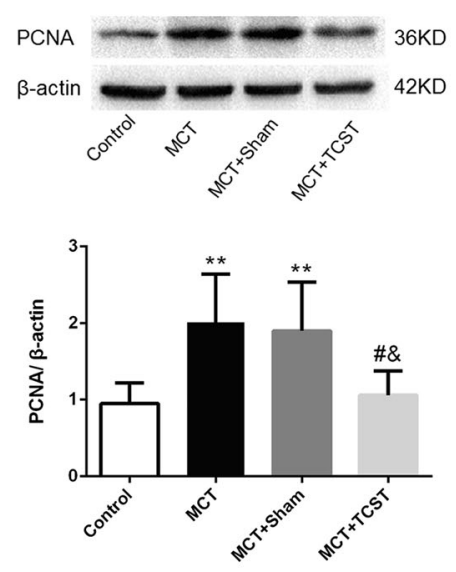

C
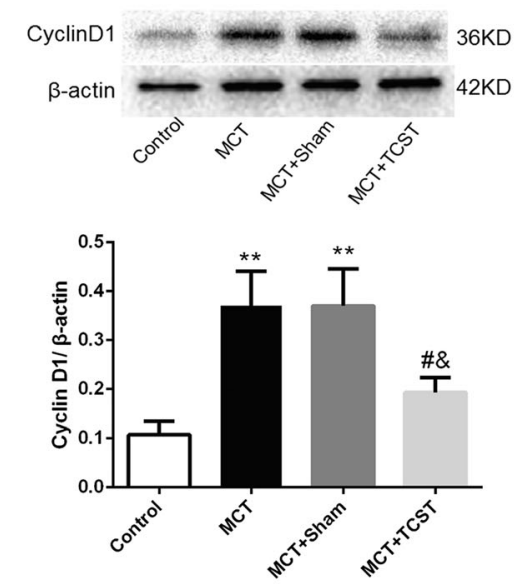

E

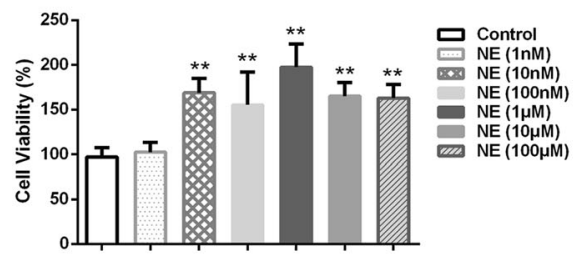

B
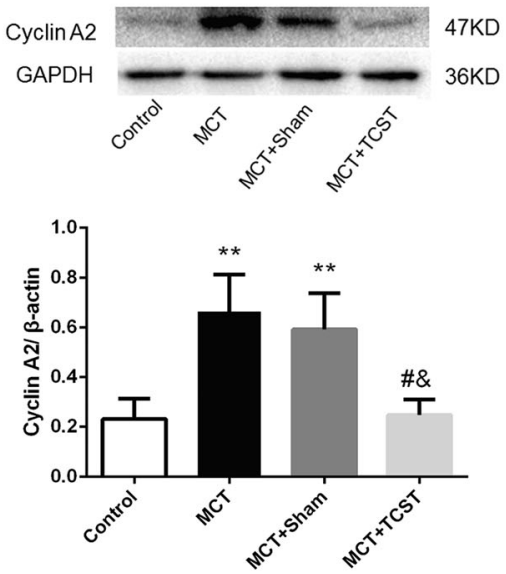

D
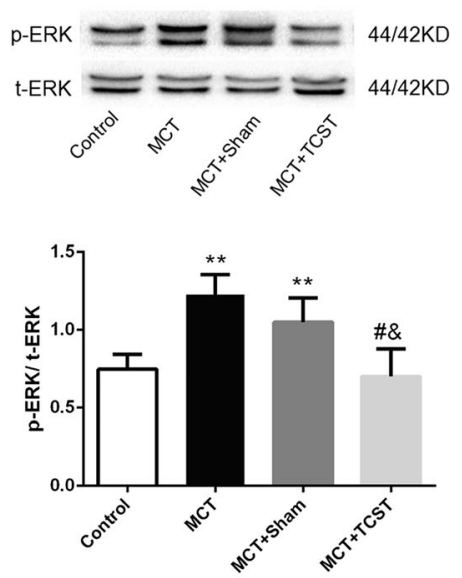

$\mathbf{F}$

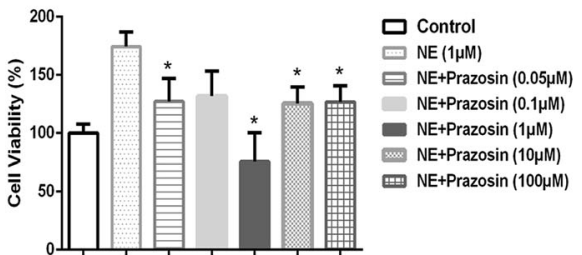

Fig. 5 TCST attenuated MCT-induced proliferation and increased cyclin protein expression in vivo. a TCST decreased the upregulated expression of PCNA induced by MCT. b, $\mathbf{c}$ The upregulated expression of cyclin A2 and cyclin D1 induced by MCT was inhibited by TCST. $\mathbf{d}$ ERK activation in the pulmonary vasculature in the different groups. ${ }^{*} P<0.01$ vs. control group; $\# P<0.01$ vs. MCT group; \&P $<0.01$ vs. MCT + sham group. e NE led to a significant increase in cell viability at a concentration of $1 \mu \mathrm{M}$. ${ }^{*} P<0.01$ vs. control group; (f) Different concentrations of Pra inhibited the NE-induced increase in cell viability. ${ }^{*} P<0.05$ vs. NE group

related to the TCST operation time, starting TCST earlier resulted in a better effect. Moreover, no significant change in the mPAP was found in control rats after TCST (Additional file 1). The present study showed that TCST attenuated the RVSP, mPAP, RV hypertrophy, and pulmonary vascular remodelling in the MCT-induced PAH rats. In addition, no negative effect on the HR and systemic blood pressure was observed in the experimental rats undergoing TCST. Na et al. reported that a left cervical ganglion block could be used to treat PAH by increasing the availability of nitric oxide [30], but there are several limitations to their results as follows: 1 ) the mPAP was not measured, 2) due to the predominance of the right stellate ganglion in the sympathetic control of the heart [31], it is better to block the right superior cervical ganglion than the left, and 3) according to our experience, 


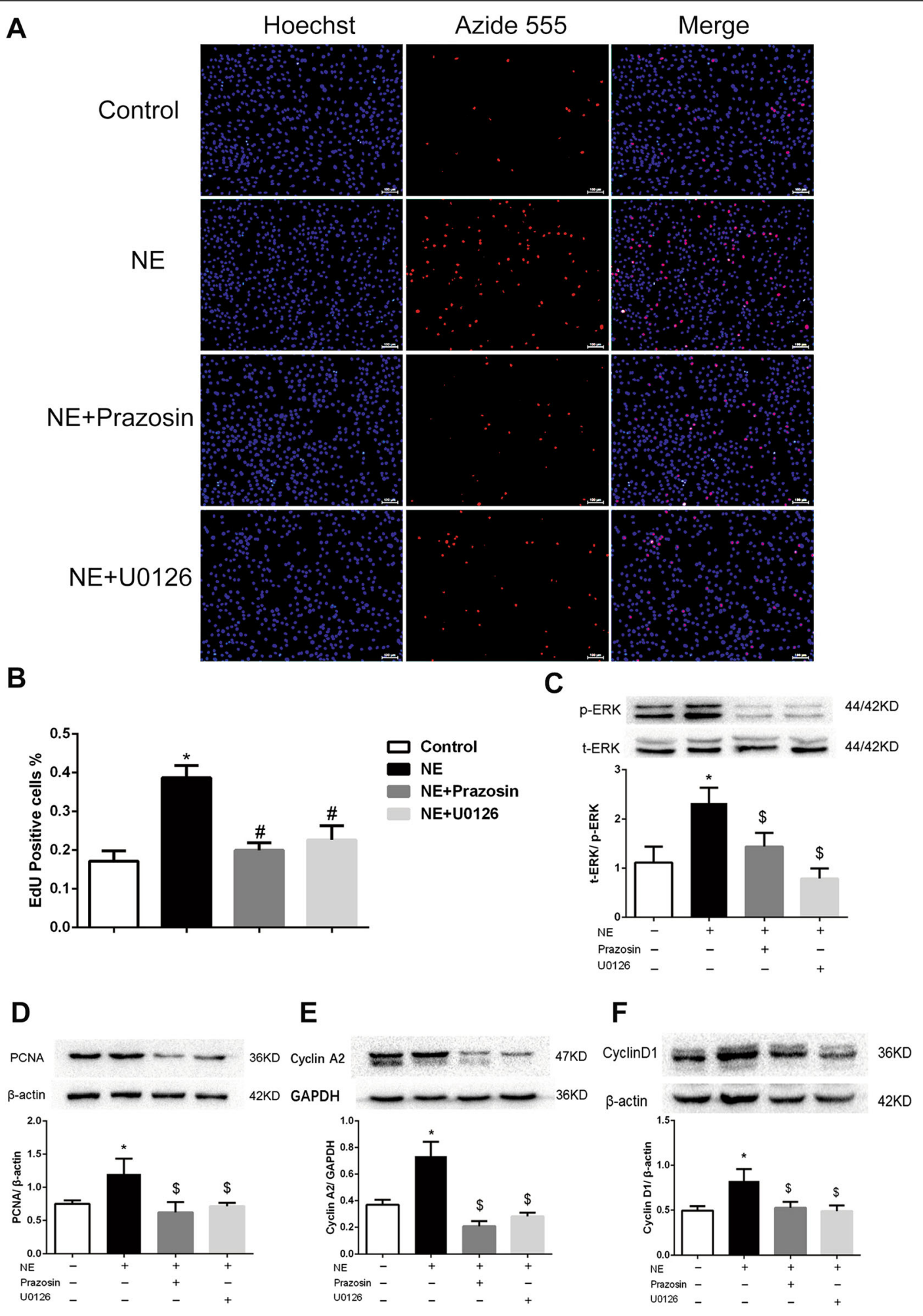

Fig. 6 (See legend on next page.) 
(See figure on previous page.)

Fig. 6 Effect of ERK-1/2 on NE-induced proliferation in PASMCS. a PASMC cell proliferation was measured by an EdU staining assay (scale bar $=100 \mu \mathrm{m})$. b NE increased the percentage of EdU-positive PASMCs. However, this effect was suppressed by the treatment with Pra or U0126. c NE increased ERK-1/2 phosphorylation in the PASMCs, and this increase was inhibited by Pra and U0126. d, e, f NE increased the expression of PCNA, cyclin A2 and cyclin D1 in the PASMCs, whereas Pra and U0126 attenuated these effects. ${ }^{*} P<0.05$ vs. control group; $\$ P<0.05$ vs. NE group

it is too late to block the sympathetic nerve starting at day 14 after the MCT injection (Additional file 1).

TCST also inhibits RV remodelling. The RVTD, which is a mid-cavitary diameter measured on an apical 4chamber view, is the most practical linear diameter used in clinical practice. The upper normal limit of the RVTD in humans is $35 \mathrm{~mm}$ [32]. The TAPSE is always used for the initial diagnosis of RV function due to its reliability and sensitivity. Among 63 patients with PAH, Forfia et al. found that for each 1-mm decrease in the TAPSE, the risk of death increased by $17 \%$ [33]. Similar results were obtained by Ghio et al. [34]. The results of the present study showed that the changes in the RVTD in each group of rats were consistent with the changes in the mPAP, and the change in the TAPSE was inversely proportional to the change in the MPAP.

A series of studies reported that the systemic sympathetic nervous system is activated in PAH patients and that NE results in adrenergic receptor-mediated vasoconstriction, dysregulated proliferation, and fibrosis through different signalling mechanisms [35]. Studies have shown that plasma venous NE levels are elevated and inversely correlated with $\mathrm{CO}$ in $\mathrm{PAH}$ patients $[7,36]$. In the present study, we found that the concentrations of NE in lung tissues of MCT rats were significantly higher than those in the controls, leading to hypertrophy of the pulmonary artery, decreased $\mathrm{CO}$. These findings were consistent with previous reports indicating that NE may aggravate heart failure and pulmonary artery remodelling [21, 37]. More importantly, the findings demonstrate that the decrease in the lumen area and the increase of the pulmonary arteries in MCT-induced PAH were inhibited by TCST. Furthermore, the accumulation of $\mathrm{T}$ cells was observed around the lung blood vessels in the MCT group, implying that MCT leads to inflammatory responses in the lung in rats. However, this phenomenon was significantly alleviated by TCST, indicating that TCST effectively inhibited MCTinduced lung inflammation. One explanation could be that TCST reduced the concentration of NE, which binds adrenergic receptors on immune cells in the PAH rats, inhibiting the consequent response [38]. These findings support the hypothesis that TCST can reduce the release of NE and inhibit PAH progression.

MCT is a toxic alkaloid that can induce PAH via endothelial damage and PASMC proliferation. The proliferation of PASMCs has been closely linked to pulmonary arterial remodelling, which is the main feature of $\mathrm{PAH}$.
In addition, the cell cycle plays a central role in cell proliferation and consists of the following distinct stages: G1, S, G2 and M phases; the progression of the cell cycle is precisely controlled and coordinated by a series of cyclins. Cyclin D1, which is expressed in numerous tissues, is a critical target of proliferative signals during the G1 phase and plays a crucial role in the G1/S transition [39]. The overexpression of cyclin D1 is known to be positively associated with the pulmonary vessel WT in rats and is correlated with the early onset of cancer $[40,41]$. Studies have shown that cyclin A2 is a target of oncogenic signals; in complex with cdk1, cyclin A2 promotes entry into mitosis [42]. In addition, the upregulated expression of cyclin A2 leads to PASMC proliferation [43]. The results of the present study show that MCT increased the expression of cyclin D1 and cyclin A2 in vivo, suggesting that $\mathrm{MCT}$ promoted cell cycle progression. However, these trends were inhibited by TCST.

We further explored the mechanism of action of NE in the process of PASMCs proliferation. ERK-1/2 is a member of the MAPK family that plays an important role in multiple cellular functions, including growth and differentiation. Previous studies have indicated that increasing proliferation in PASMCs is a part of the sustained activation of ERK-1/2 and that the inhibition of ERK-1/2 activation may be a therapeutic target in PAH [21, 43, 44]. Based on the results of the present study, the phosphorylation of ERK-1/2 was significantly increased in the pulmonary arteries of the MCT-treated rats, which was consistent with the change in NE. In vitro, NE promoted PASMC proliferation at the optimal concentration of $1 \mu \mathrm{M}$ and increased the phosphorylation of ERK-1/2 and proliferation markers, such as PCNA, cyclin D1 and cyclin A2. Moreover, ERK-1/2 phosphorylation was abolished by the treatment with Pra, and the expression of the proliferation markers was prevented by U0126 (a specific inhibitor of ERK-1/2). These results suggest that NE facilitates PASMC proliferation partially via the activation of the ERK-1/2 signalling pathway.

Sympathetic activation may be a compensatory response that improves cardiac function during the early stages, but marked sympathetic hyperactivity aggravates the progression of heart failure. In addition, RV failure is one of the pathologies of $\mathrm{PAH}$, and reduced parasympathetic nervous activation is associated with disease progression in $\mathrm{PAH}$ [45]. Although some studies suggest that drugs that depress 
sympathetic nervous activity, such as adrenergic antagonists, could be used to treat $\mathrm{PAH}$, the value of vasodilatory treatment in $\mathrm{PAH}$ is still controversial [21, 46, 47]. First, because $\alpha 1$-adrenergic receptors are expressed in pulmonary vascular tissue at density levels similar to resistance blood vessels, most $\alpha$ adrenergic antagonists usually result in systemic hypotension. Early $\alpha$-adrenergic antagonists were used to treat $\mathrm{PAH}$ included prazosin and phentolamine; however, the clinical application of such drugs is restricted due to their side effects, including dyspnoea, systemic hypotension, and adverse haemodynamic effects $[48,49]$. Second, $\beta$-adrenergic antagonists have recently been considered a therapeutic strategy for PAH, and some small observational studies have reported that $90 \%$ of patients demonstrated significant improvement in the 6-min walk distance test following $\beta$-blocker treatment [50]. However, some experts have reservations regarding the safety profile of $\beta$-blockers. A clinical study reported that bisoprolol produced no improvement in the $\mathrm{RV}$ ejection fraction but resulted in a decrease in both the HR and cardiac index and exhibited a trend towards reduced exercise capacity [51]. The present study demonstrated that TCST improved the haemodynamics, pulmonary artery remodelling, and RV function in MCTinduced PAH rats. Additionally, one advantage of TCST is that this treatment has no effect on systemic blood pressure, HR or CO. Thus, adrenergic antagonists may not be an alternative for TCST.

This study has several limitations. First, sympathetic reinnervation has been shown to occur in a previously denervated rat lung [52]. However, Horner's syndrome in rats did not disappear during our study, indicating that the sympathetic reinnervation was not complete. Second, there was an absence of histological evidence of pulmonary sympathetic nerves, demonstrating a lack of histological proof of sympathetic nerve changes after TCST. Third, whether the other two signalling pathways of the MAPK family, i.e., JNK and P38, are also involved in mediating the TCST-induced attenuation of the progression of PAH was not determined.

\section{Conclusion}

The present study demonstrates that TCST is an effective and specific method of sympathetic denervation that does not affect systemic blood pressure or cause injury to the blood vessels and parasympathetic nerves. Moreover, TCST inhibited the MCT-induced progression of $\mathrm{PAH}$ in rats likely by decreasing the NE concentrations in the lung tissues and thereby inhibiting PASMCs proliferation mediated by the ERK-1/2 signalling pathway. We provided novel insight into the use of this nonpharmacologic strategy in patients with PAH.

\section{Additional file}

\begin{abstract}
Additional file 1: Figure legends (A) To determine the optimal time to intervene, we have conducted a preliminary experiment. Different timepoint of TCST were checked and compared in the MCT rats as follows: the same day (D0), the third day (D3), the seventh day (D7) and the fourteenth day (D14) after MCT injection, respectively. Then the pulmonary arterial pressure was measured by right heart catheterization on 21 day (D21) after MCT administration. (B) Our results found the rats with lowest MPAP was that undergoing TCST on the same day of MCT injection among treatment groups. But no significant decrease of mPAP was found in rats when the TCST time started on 14th day. Besides, there was no significant change in MPAP in control +TCST group. As a result, the prognosis of rats with PAH was related with TCST operational time, the earlier TCST started, the better effect was. ${ }^{*} P<0.01$ vs. control group; $\# \mathrm{P}<0.01$ vs. MCT group. (C) Cardiac output (CO) was measured on M-mode echocardiography parasternal long-axis views. (D) Pulmonary artery transverse diameter (PATD) were measured on parasternal short-axis views. (E) Right ventricular transverse diameter (RVTD) were measured on apical 4chamber view. AO, aorta; LA, left atrium; LV, left ventricle; PA, pulmonary artery; RV, right ventricle. (TIF $9101 \mathrm{~kb}$ )
\end{abstract}

\section{Abbreviations}

CO: Cardiac output; ERK: Extracellular signal-regulated kinase; HR: Heart rate; IP: Intraperitoneal injection; LV: Left ventricle; MCT: Monocrotaline; mPAP: Mean pulmonary arterial pressure; mSAP: Mean systemic arterial pressure; NE: Norepinephrine; PADN: Pulmonary artery denervation; PAH: Pulmonary arterial hypertension; PASMCs: Pulmonary arterial smooth muscle cells; PATD: Pulmonary artery transverse diameter; PCNA: Proliferating cell nuclear antigen; Pra: Prazosin; RV: Right ventricular; RVSP: Right ventricular systolic pressure; RVTD: Right ventricular transverse diameter; S: Septum; SGBs: Stellate ganglion blocks; TAPSE: Tricuspid annular plane systolic excursion; TCST: Transection of the cervical sympathetic trunk; TH: Tyrosine hydroxylase; WT: Medial wall thickness; a-SMA: a-smooth muscle actin

\section{Acknowledgements}

Not applicable.

\section{Authors' contributions}

ZYP, XR, QS, XL and HW conceived the idea and designed the experiment. $Z Y P, P X, Y G Q$ and DQ performed the experiments and completed the analysis. ZYP, PX, XR, YGQ and DQ conducted the data interpretation and prepared the manuscript. HW, QS and XL critically revised the manuscript. All authors have provided final approval of the version to be published.

\section{Funding}

This study was supported by the National Natural Science Foundation of China [81170188 and 30971212], the Natural Science Foundation of Chongqing [CSCT2009BB5069], and the Chongqing Municipal Health and Family Planning Commission [2016HBRC001 and 2016XMSB0003767].

\section{Availability of data and materials}

The datasets used and/or analysed during the current study are available from the corresponding author upon reasonable request.

Ethics approval and consent to participate

This study was approved by the Ethics Committee of Chongqing Medical University (Chongqing, China).

\section{Consent for publication}

Not applicable.

\section{Competing interests}

The authors declare that they have no competing interests.

\section{Author details}

${ }^{1}$ Department of Cardiology, the First Affiliated Hospital of Chongqing Medical University, 1 Youyi Road, Yuzhong District, Chongqing, China. ${ }^{2}$ Department of Medicine, Section of Pulmonary, Critical Care, Sleep and 
Allergy, University of Illinois at Chicago, Chicago, IL, USA. ${ }^{3}$ Present Address: Lung Vascular Biology Program, NHLBI/NIH, Bethesda, MD, USA.

\section{Received: 20 January 2019 Accepted: 3 June 2019} Published online: 14 June 2019

\section{References}

1. Galie N, Humbert M, Vachiery JL, Gibbs S, Lang I, Torbicki A, et al. 2015 ESC/ ERS guidelines for the diagnosis and treatment of pulmonary hypertension: the joint task force for the diagnosis and treatment of pulmonary hypertension of the European Society of Cardiology (ESC) and the European Respiratory Society (ERS): endorsed by: Association for European Paediatric and Congenital Cardiology (AEPC), International Society for Heart and Lung Transplantation (ISHLT). Eur Heart J. 2016;37(1):67-119.

2. Maron BA, Loscalzo J. Pulmonary hypertension: pathophysiology and signaling pathways. Handb Exp Pharmacol. 2013;218:31-58.

3. Schermuly RT, Ghofrani HA, Wilkins MR, Grimminger F. Mechanisms of disease: pulmonary arterial hypertension. Nat Rev Cardiol. 2011;8(8):443-55

4. Farber HW, Miller DP, Poms AD, Badesch DB, Frost AE, Muros-Le Rouzic E, et al. Five-year outcomes of patients enrolled in the REVEAL registry. Chest. 2015;148(4):1043-54

5. Benza RL, Miller DP, Barst RJ, Badesch DB, Frost AE, McGoon MD. An evaluation of long-term survival from time of diagnosis in pulmonary arterial hypertension from the REVEAL registry. Chest. 2012;142(2):448-56.

6. Mak S, Witte KK, Al-Hesayen A, Granton JJ, Parker JD. Cardiac sympathetic activation in patients with pulmonary arterial hypertension. Am J Physiol Regul Integr Comp Physiol. 2012;302(10):R1153-7.

7. Velez-Roa S, Ciarka A, Najem B, Vachiery JL, Naeije R, van de Borne P. Increased sympathetic nerve activity in pulmonary artery hypertension. Circulation. 2004:110(10):1308-12.

8. Ciarka A, Doan V, Velez-Roa S, Naeije R, van de Borne P. Prognostic significance of sympathetic nervous system activation in pulmonary arterial hypertension. Am J Respir Crit Care Med. 2010;181(11):1269-75.

9. Wensel R, Jilek C, Dorr M, Francis DP, Stadler H, Lange T, et al. Impaired cardiac autonomic control relates to disease severity in pulmonary hypertension. Eur Respir J. 2009;34(4):895-901.

10. Qingyan Z, Xuejun J, Yanhong T, Zixuan D, Xiaozhan W, Xule W, et al. Beneficial effects of renal denervation on pulmonary vascular remodeling in experimental pulmonary artery hypertension. Rev Esp Cardiol (Engl Ed). 2015;68(7):562-70.

11. Hu W, Yu SB, Chen L, Guo RQ, Zhao QY. Renal sympathetic denervation prevents the development of pulmonary arterial hypertension and cardiac dysfunction in dogs. Kaohsiung J Med Sci. 2015;31(8):405-12.

12. Chen SL, Zhang FF, Xu J, Xie DJ, Zhou L, Nguyen T, et al. Pulmonary artery denervation to treat pulmonary arterial hypertension: the single-center, prospective, first-in-man PADN-1 study (first-in-man pulmonary artery denervation for treatment of pulmonary artery hypertension). J Am Coll Cardiol. 2013;62(12):1092-100

13. Zhou L, Zhang J, Jiang XM, Xie DJ, Wang JS, Li L, et al. Pulmonary artery denervation attenuates pulmonary arterial remodeling in dogs with pulmonary arterial hypertension induced by dehydrogenized Monocrotaline. JACC Cardiovasc Interv. 2015;8(15):2013-23.

14. Liu C, Jiang XM, Zhang J, Li B, Li J, Xie DJ, et al. Pulmonary artery denervation improves pulmonary arterial hypertension induced right ventricular dysfunction by modulating the local renin-angiotensinaldosterone system. BMC Cardiovasc Disord. 2016;16(1):192.

15. Zhang $H$, Zhang J, Xie DJ, Jiang X, Zhang FF, Chen SL. Pulmonary artery denervation for treatment of a patient with pulmonary hypertension secondary to left heart disease. Pulm Circ. 2016:6(2):240-3.

16. Demir A, Dönmez A, Erdemli Ö. Stellate ganglion blockade in coronary surgery. Anestezi Dergisi. 2011;19(4):201-7.

17. Fudim M, Boortz-Marx R, Patel CB, Sun AY, Piccini JP. Autonomic modulation for the treatment of ventricular arrhythmias: therapeutic use of percutaneous stellate ganglion blocks. J Cardiovasc Electrophysiol. 2017;28(4):446-9.

18. Leftheriotis D, Flevari P, Kossyvakis C, Katsaras D, Batistaki C, Arvaniti C, et al. Acute effects of unilateral temporary stellate ganglion block on human atrial electrophysiological properties and atrial fibrillation inducibility. Heart Rhythm. 2016;13(11):2111-7.

19. Elias M. Cervical sympathetic and stellate ganglion blocks. Pain Physician. 2000;3(3):294-304.
20. Kummer W. Pulmonary vascular innervation and its role in responses to hypoxia: size matters! Proc Am Thorac Soc. 2011;8(6):471-6.

21. Liu R, Zhang Q, Luo Q, Qiao H, Wang P, Yu J, et al. Norepinephrine stimulation of alpha1D-adrenoceptor promotes proliferation of pulmonary artery smooth muscle cells via ERK-1/2 signaling. Int J Biochem Cell Biol. 2017;88:100-12.

22. Faber JE, Szymeczek CL, Cotecchia S, Thomas SA, Tanoue A, Tsujimoto G, et al. Alpha1-adrenoceptor-dependent vascular hypertrophy and remodeling in murine hypoxic pulmonary hypertension. Am J Physiol Heart Circ Physiol. 2007:292(5):H2316-23.

23. Xiao L, Pimentel DR, Wang J, Singh K, Colucci WS, Sawyer DB. Role of reactive oxygen species and NAD(P) H oxidase in a1-adrenoceptor signaling in adult rat cardiac myocytes. Am J Physiol Cell Physiol. 2002;282(4):C926-34.

24. Agbani EO, Coats P, Mills A, Wadsworth RM. Peroxynitrite stimulates pulmonary artery endothelial and smooth muscle cell proliferation: involvement of ERK and PKC. Pulm Pharmacol Ther. 2011:24(1):100-9.

25. Savastano LE, Castro AE, Fitt MR, Rath MF, Romeo HE, Muñoz EM. A standardized surgical technique for rat superior cervical ganglionectomy. J Neurosci Methods. 2010;192(1):22-33.

26. Crystal RG, Ronald G. The lung: scientific foundations. JAMA: LippincottRaven; 1997.

27. Richardson JB. Nerve supply to the lungs. Am Rev Respir Dis. 1979;119(5):785.

28. Townsley MI. Structure and composition of pulmonary arteries, capillaries, and veins. Compr Physiol. 2012;2(1):675-709.

29. Barnes PJ, Liu SF. Regulation of pulmonary vascular tone. Pharmacol Rev. 1995:47(1):87

30. Na S, Kim OS, Ryoo S, Kweon TD, Choi YS, Shim HS, et al. Cervical ganglion block attenuates the progression of pulmonary hypertension via nitric oxide and arginase pathways. Hypertension. 2014;63(2):309-15.

31. Yokota S, Taneyama C, Goto H. Different effects of right and left stellate ganglion block on systolic blood pressure and heart rate. Open J Anesthesiol. 2013;03(03):143-7.

32. Kossaify A. Echocardiographic assessment of the right ventricle, from the conventional approach to speckle tracking and three-dimensional imaging, and insights into the "right way" to explore the forgotten chamber. Clin Med Insights Cardiol. 2015;9:65-75.

33. Forfia PR, Fisher MR, Mathai SC, Housten-Harris T, Hemnes AR, Borlaug BA, et al. Tricuspid annular displacement predicts survival in pulmonary hypertension. Am J Respir Crit Care Med. 2006:174(9):1034-41.

34. Ghio S, Temporelli PL, Klersy C, Simioniuc A, Girardi B, Scelsi L, et al. Prognostic relevance of a non-invasive evaluation of right ventricular function and pulmonary artery pressure in patients with chronic heart failure. Eur J Heart Fail. 2013;15(4):408-14.

35. Maron BA, Leopold JA. Emerging concepts in the molecular basis of pulmonary arterial hypertension: part II: Neurohormonal signaling contributes to the pulmonary vascular and right ventricular Pathophenotype of pulmonary arterial hypertension. Circulation. 2015;131(23):2079-91.

36. Nagaya $\mathrm{N}$, et al. Plasma brain natriuretic peptide as a prognostic indicator in patients with primary pulmonary hypertension. Circulation. 2000;8(102).

37. Xiao L, Pimental DR, Amin JK, Singh K, Sawyer DB, Colucci WS. MEK1/2ERK1/2 mediates alpha1-adrenergic receptor-stimulated hypertrophy in adult rat ventricular myocytes. J Mol Cell Cardiol. 2001;33(4):779-87.

38. Slota C, Shi A, Chen G, Bevans M, Weng NP. Norepinephrine preferentially modulates memory CD8 T cell function inducing inflammatory cytokine production and reducing proliferation in response to activation. Brain Behav Immun. 2015:46:168-79.

39. Fu M, Wang CZ, Sakamaki T, Pestell RG. Minireview: cyclin D1: normal and abnormal functions. Endocrinology. 2004;145(12):5439-47.

40. Igarashi K, Masaki T, Shiratori Y, Rengifo W, Nagata T, Hara K, et al. Activation of cyclin D1-related kinase in human lung adenocarcinoma. $\mathrm{Br} J$ Cancer. 1999:81(4):705.

41. Zhou S, Li M, Zeng D, Sun G, Zhou J, Wang R. Effects of basic fibroblast growth factor and cyclin D1 on cigarette smoke-induced pulmonary vascular remodeling in rats. Exp Ther Med. 2015;9(1):33-8.

42. Pagano $M_{1}$., Pepperkok $R$, ., Verde $F$, ., Ansorge $W_{1}$., Draetta $G$, . Cyclin a is required at two points in the human cell cycle. EMBO J 1992, 11(3):961-971.

43. Li M, Liu Y, Sun $X$, Li Z, Liu Y, Fang $P$, et al. Sildenafil inhibits calcineurin/ NFATc2-mediated cyclin a expression in pulmonary artery smooth muscle cells. Life Sci. 2011;89(17-18):644-9.

44. Gao H, Cheng Y, Zong L, Huang L, Qiao C, Li W, et al. Aspirin attenuates monocrotaline-induced pulmonary arterial hypertension in rats by suppressing the ERK/MAPK pathway. Clin Exp Hypertens. 2017;39(1):34-41. 
45. da Silva Goncalves Bos D, CEE VDB, Kurakula K, Sun XQ, Casali KR, Casali AG, et al. Contribution of impaired parasympathetic activity to right ventricular dysfunction and pulmonary vascular remodeling in pulmonary arterial hypertension. Circulation. 2018:137(9):910-24.

46. Ishikawa M, Sato N, Asai K, Takano T, Mizuno K. Effects of a pure alpha/betaadrenergic receptor blocker on monocrotaline-induced pulmonary arterial hypertension with right ventricular hypertrophy in rats. Circ J. 2009;73(12):2337-41.

47. Okumura K, Kato H, Honjo O, Breitling S, Kuebler WM, Sun M, et al. Carvedilol improves biventricular fibrosis and function in experimental pulmonary hypertension. J Mol Med. 2015;93(6):663-74.

48. Cohen ML, Kronzon I. Adverse hemodynamic effects of phentolamine in primary pulmonary hypertension. Ann Intern Med. 1981;95(5):591-2.

49. Heinrich U, Angehrn W, Steinbrunn W. Therapy of primary pulmonary hypertension with phentolamine. Schweizerische Medizinische Wochenschrift. 1983;113(4):145.

50. Provencher S, Herve $P$, Jais $X$, Lebrec $D$, Humbert M, Simonneau G, et al. Deleterious effects of beta-blockers on exercise capacity and hemodynamics in patients with portopulmonary hypertension. Gastroenterology. 2006;130(1):120-6.

51. van Campen JS, De BK, MC VDV, Ce VDB, Allaart CP, Raijmakers PG, et al. Bisoprolol in idiopathic pulmonary arterial hypertension: an explorative study. Eur Respir J. 2016;48(3):787-96.

52. Takachi T, Maeda M, Shirakusa T, Hayashida Y. Sympathetic reinnervation of unilaterally denervated rat lung. Acta Physiologica 2010, 154(1):43-50.

\section{Publisher's Note}

Springer Nature remains neutral with regard to jurisdictional claims in published maps and institutional affiliations.

\section{Ready to submit your research? Choose BMC and benefit from:}

- fast, convenient online submission

- thorough peer review by experienced researchers in your field

- rapid publication on acceptance

- support for research data, including large and complex data types

- gold Open Access which fosters wider collaboration and increased citations

- maximum visibility for your research: over $100 \mathrm{M}$ website views per year

At $\mathrm{BMC}$, research is always in progress.

Learn more biomedcentral.com/submissions 